\title{
A finite element method for compressible and incompressible flows
}

\author{
Nacer E. El Kadri E. ${ }^{1}$. Abdelhakim Chillali ${ }^{1}$ (1)
}

Received: 18 October 2019 / Accepted: 18 December 2019 / Published online: 2 January 2020

(c) Springer Nature Switzerland AG 2020

\begin{abstract}
In this study, we present a unified formulation of compressible and incompressible Navier-Stokes equations in the quasi-linear form for primitive variables. In this formulation, two thermodynamic parameters, coefficient of isothermal compressibility and coefficient of thermal expansion, are highlighted. The incompressible limit is obtained when the coefficients of isothermal compressibility and of thermal expansion are taken equal to zero and when the density is supposed constant. For the simulation of advection-dominated flows, a stabilized finite element method based on the Petrov-Galerkin formulation is proposed. The proposed unified formulation is tested and validated for different numerical simulations. Different test cases are processed, from simplified models to more elaborate models. Finally, we present the conclusions inspired by this work, as well as the perspectives envisaged.
\end{abstract}

Keywords Navier-Stokes · Compressible · Incompressible · Finite element method · Petrov-Galerkin

\section{Introduction}

The laws of fluid mechanics are now well established. They were derived from Navier in 1827, Poisson in 1831, Saint-Venant in 1843 and Stokes in 1845. They express the conservation of mass, momentum (Newton's second law) and energy (first principle of thermodynamics). Whatever the complexity of the physical phenomena involved, these principles of conservation not only determine the evolution of any system at any time, but are also sufficient to describe it. To these conservation laws, we must add a description of the fluid (law of behaviour), which can be analytic or parametric.

Since the 1970s, modelling and numerical simulation has become increasingly important in design and design processes in a large number of industrial sectors. Examples include aeronautics, hydrodynamics, acoustics, combustion, thermodynamics, and electromagnetism. Current challenges lie in improving performance in each of these sectors, but also in the coupling of different physics, of which aero-elasticity is a good example. The goal is of course to minimize costs compared to experimental testing by reducing the number of models or configurations tested by previous numerical studies. The combined use of experimental means and numerical tools makes the design process more reliable and improves performance by reducing the margins of error. The development of numerical simulations is of course very closely linked to the continuous development of computer tools and computer machines. Nevertheless, despite the incredible power of some current machines, their performance is still very far from being able to directly solve some problems occurring in the sector. This is why, in addition to a part of the numerical modelling (solving a physical problem described in the form of equations, using computers) often limited by the power of computing machines, is added a part physical modelling (simplification of the problem by physical or mathematical considerations). From an academic point of view, these two aspects (numerical modelling and physical modelling) continue to progress jointly today and still raise many questions (need to stabilize the

$\triangle$ Abdelhakim Chillali, abdelhakim.chillali@usmba.ac.ma | ${ }^{1}$ Engineering Sciences Laboratory - LSI, Polydisciplinary Faculty of Taza, Sidi Mohamed Ben Abdellah University, Taza, Morocco. 
numerical methods, more and more complex in turbulent modelling...).

In the field of computational fluid dynamics (CFD), which is of particular interest to us in this work, numerical simulation is a promising tool for understanding flow physics. This allows for example to obtain information at any point in space, which is not conceivable experimentally. In addition, the constant increase in the power of computer machines makes it possible to consider the simulation of increasingly complex geometries with increasingly complex solutions. However, to get relevant information about a flow, you need a numerical scheme with good properties. The first essential properties are stability and consistency. The stability of a scheme reflects its ability to adopt a regular behaviour when introducing a disturbance due, for example, to the incorrect calculation of a boundary condition or an initial condition. A scheme is said to be stable if any disturbance of numerical origin is attenuated or, at best, unamplified. We will say that it is unstable if, on the contrary, a disturbance, even a small one, is amplified in time or in space. A numerical scheme is said to be coherent, if and only if, the discretized formulation of the partial differential equations tends towards the continuous formulation, when all the stages of the discretization tend towards zero. In addition to its stability and consistency, the accuracy of a numerical scheme is also a primary property. This is the mathematical distance from the numerical solution to the exact solution. It is well known that this quantity is often of the order of a power of the size characteristic of the mesh used. This power is called the order of the method. It is also a measure of the speed of convergence of the method.

The most widely used numerical methods in the field of fluid mechanics are finite volume and finite element methods. Finite volume methods are undoubtedly one of the best known and best documented methods. This is because most industrial fluid mechanics software has been built from these methods. Finite element methods, initially designed to deal with symmetric and coercive problems, as is the case in deformable media mechanics, finite elements were quickly used outside of this context to solve fluid mechanics problems. Finite element methods have the advantage of being less expensive and more efficient than finite volume methods for high order, since the compactness of the scheme is preserved as the order of this scheme increases. Currently, the finite element method is one of the most powerful numerical methods designed to date. The characteristics that have led to its popularity include: the ease of modelling complex shape geometries, the natural processing of boundary conditions of differential type, as well as the possibility of being programmed in the form of software, software adaptable to treatment of a wide range of problems. These advantages, together with personal reasons for continuity of research, led us to choose the finite element method.

The classical formulation of the finite element method is based on the Galerkin weighted residue method. This method consists in choosing as weighting functions the interpolation functions retained for the description of the local field of the unknown variable of the problem. The numerical resolution of the equations of fluid mechanics (convection-diffusion equations) by classical finite element methods (Galerkin) encounters a major difficulty: a numerical instability appears which finds its origin in the convective term. This problem is also encountered by finite difference or finite volume methods. The discretization of the convective term by the Galerkin method produces a system of non-symmetric equations that are decoupled from one node to another. In this case, if the diffusive term is very small in front of the term convection, the solution may become unstable in areas of high gradient (boundary layer, mixing layer, and shock wave). By "instability" is meant either the appearance of oscillations in the solution obtained by a direct inversion method, or the lack of convergence when using an iterative method. A traditional remedy to this problem is to add an artificial diffusion in order to stabilize the numerical scheme. However, in the general case (multidimensional, unsteady, and with the source term) the addition of an artificial diffusion is not obvious and precautions must be taken to preserve the precision of the numerical scheme. Thus, in the field of computational fluid dynamics, since the 1980s, stable finite element formulations have appeared. The principle of these methods is to add a stabilization term to the Galerkin formulation. This term is chosen so as to preserve the consistency and order of the scheme. At first the stabilized finite element methods have been developed for convection-diffusion equations [1], as well as for the Stokes problem. Subsequently they have been generalized to the incompressible [2,3] and compressible Navier-Stokes equations [4]. Stabilized finite elements have been and are still the subject of much research. Thus, we can distinguish two large families of stabilized finite element methods: those of multi-scale type, and those called Petrov-Galerkin. The first category includes the following methods: finite elements of bubble type [5], Variational Multi-Scale (VMS) [6], Sub-Grid Scale (SGS) [7], Orthogonal Sub-Grid Scale (OSGS) [8], methods of internal penalization [9], while the second category groups the methods: Streamline-Upwind/Petrov-Galerkin (SUPG) [2], Pressure-Stabilization/Petrov-Galerkin (PSPG) [2, 3], Galerkin Least Square (GLS) [10], Gradient Galerkin Least Square (GGLS) [11] for example.

Without going into technical details, the principle of the Petrov-Galerkin formulation, as well as the main lines of research of which it is the subject, are presented. As 
already mentioned, a stabilized finite element approach is based on the Galerkin formulation plus a stabilization term. This amounts to moving from a Galerkin formulation to a Petrov-Galerkin formulation by modifying the weighting functions of the weak formulation. More precisely, the starting point is a partial derivative equation of the form:

$\mathcal{L} u=f$

where $\mathcal{L}$ is a differential operator. An approximation of the solution $u$, denoted $\tilde{u}$, is then sought in the approximation space of finite dimension $\left(\phi_{1}, \ldots, \phi_{N}\right), \tilde{u}=\sum_{i} u_{i} \phi_{i}$. The weak formulation associated with this problem is then written:

$$
\int_{\Omega} \mathrm{w}^{*}(\mathcal{L} \tilde{u}-f) d \Omega=0
$$

The choice $w^{*}=\phi$ leads to the Galerkin formulation, while other choices $\mathrm{w}^{*} \neq \phi$ lead to the so-called Petrov-Galerkin formulations. In stabilized finite element methods, a disturbance is added to the basic functions of the Galerkin formulation. This disturbance is of the form:

$\mathrm{w}^{*}=\mathrm{w}+\mathcal{L}_{\text {stab }}(\mathrm{w}) \tau$

where $\mathcal{L}_{\text {stab }}$ denotes a stabilization operator that differs according to the methods. For the formulation named Streamline Upwind Petrov-Galerkin (SUPG) [1], it corresponds to the convective part of the operator $\mathcal{L}$. It is also possible to define it as $\mathcal{L}_{\text {stab }}=\mathcal{L}$. In this case it is called the Galerkin Least Squares (GLS) formulation. The consistency of the formulation is ensured by the product of the residue of the equation with the term perturbation. Finally, a stabilization parameter also appears in the disturbance. This stabilization parameter, commonly noted $\tau$, makes it possible to control the amount of numerical viscosity introduced in the scheme. This parameter is constructed from flow-specific quantities such as velocity, density, pressure, and quantities of numerical discretization such as time step and mesh size. Even today, the choice of the stabilization parameter of the Petrov-Galerkin formulation remains an open problem, many formulations have been proposed but research is still ongoing on the subject. The Petrov-Galerkin formulation was first constructed to solve compressible Navier-Stokes equations using conservative variables [1]. This seems to be a natural choice because of the conservative nature of these equations. There are, however, other possible sets of variables, such as, for example, entropy variables [12]. Thanks to the use of these variables, the system is written in pseudo-linear form and becomes symmetrical. It is then possible to better understand the basic aspects of the Petrov-Galerkin formulation and to demonstrate certain properties. For example, the study of Clausius-Duhem's entropic inequality has highlighted the indispensability of an additional term of diffusion in the presence of shocks. It has been shown, however, [13-16] that in the vast majority of case the Petrov-Galerkin formulation, constructed from conservative variables, give results similar to those constructed from entropy variables. Also, [13-16] developed Petrov-Galerkin formulations for compressible flow simulation based on sets of variables other than conservative variables or entropy variables, they use primitive variables. Once again, there is no significant difference in the results obtained. Nevertheless, at the limit of the incompressible, the matrices of the system obtained with the help of the primitive variables remain limited. This set of variables thus seems to be an interesting candidate for the formulation of a method valid for both the compressible and the incompressible. Insofar as it has not been demonstrated that, for compressible flow simulation, primitive variables are more conservative than conservative variables, and since they are easier to set up in a computer code, the choice of primitive variables will be retained later. When transonic or supersonic flows are studied, the important issue is the resolution of shock waves. Now, it is important for such problems to treat the Navier-Stokes equations written in conservative form. Indeed, if the conservation of the mass, the momentum and the energy were not respected, the speed of displacement of the shock wave could be false. This is one of the main weaknesses in the use of entropy variables. The use of entropy variables has highlighted the need for an additional dissipation mechanism, for the Petrov-Galerkin method, in the presence of shock waves in a compressible flow. It is for this reason that a term called shock capture has been developed.

The numerical resolution of compressible and incompressible Navier-Stokes equations requires the use of accurate and robust numerical schemes. Historically, these schemes have been developed independently taking into account problems related to numerical stability and the choice of variables associated with flow problems. Therefore a numerical scheme dedicated to the resolution of incompressible flows is often unusable for the resolution of compressible flows and vice versa. In this case, we speak of non-unified schema. To solve this problem, several researchers have proposed numerical schemes for a unified approach to compressible and incompressible flows. Weiss and Smith [17], Turkel [18], Guillard and Murrone [19], in the context of low Mach flows, proposed to use the numerical methods dedicated to the resolution of compressible systems by repackaging the initial system. Yoon and Yabe [20], Ida [21], Xiao et al. [22] extended projection methods Harlow and Welch [23] and Chorin [24] for the resolution of equations of incompressible flows at the resolution of compressible flow equations. Hauke 
and Hughes [25, 26], Polner et al. [27], they presented a unified finite element method for solving the compressible and incompressible Navier-Stokes equations, to do this the equations are written in a unified way for different variables associated with flow problems. Pesch et al. [28] proposed a unified Discontinuous Galerkin method for solving compressible and incompressible Navier-Stokes equations. Other very interesting works have also been done, see for example Bijl and Wesseling [29], Caiden et al. [30], Nithiarasu et al. [31]

This paper is based on the detailed presentation of the tools used in this work, namely the unified formulation of compressible and incompressible Navier-Stokes equations and their discretization by a stabilized finite element method. A first part is devoted to the presentation of the unified formulation of compressible and incompressible Navier-Stokes equations necessary for the design of a Petrov-Galerkin formulation valid for compressible and incompressible Navier-Stokes equations. To begin, we recall the conservative and quasi-linear forms of compressible Navier-Stokes equations, for different sets of unknowns, conservative and primitive. In order to close this system, a perfect gas state law is employed. In these various formulations, two thermodynamic parameters (coefficient of isothermal compressibility and coefficient of thermal expansion) are highlighted. They allow in particular passing to the incompressible limit in a rather natural way for the primitive unknowns, the matrices of the system obtained with the help of the primitive variables remain bounded. This set of primitive variables is interesting for the formulation of a valid method for both the compressible and the incompressible, in addition the primitive variables are easier to set up in a computation code, the choice of the primitive variables will be retained later. A second part is dedicated to the presentation of the stabilized finite element method for compressible and incompressible Navier-Stokes equations written in unified form. However, the Galerkin formulation applied to the unified form of Navier-Stokes equations gives rise to an unstable numerical scheme for two reasons. On the one hand, when the convection terms of the Navier-Stokes equations are dominant, on the other hand if the discrete spaces for velocity and pressure do not satisfy the condition of inf-sup stability. To circumvent these two difficulties, it is necessary to introduce a stabilization term in the Galerkin formulation. The Petrov-Galerkin methods are detailed here, including the SUPG and GLS stabilizations. Finally, we present the conclusions inspired by this work, as well as the perspectives envisaged.

\section{Compressible Navier-Stokes equations}

The compressible Navier-Stokes equations, in conservative form, are written in vector form:

$\mathbf{U}_{, t}+\mathbf{F}_{i, i}^{\text {conv }}(\mathbf{U})=\mathbf{F}_{i, i}^{\text {diff }}(\mathbf{U})+\mathcal{F}(\mathbf{U})$

where $\mathbf{U} \in \mathbb{R}^{5}$ is the vector of the conservative variables, $\mathbf{F}_{i}^{\text {conv }}, \mathbf{F}_{i}^{\text {diff }} \in \mathbb{R}^{5}$ are respectively the convective and diffusive fluxes in the ith direction, and $\mathcal{F}$ the vector of the external forces:

$$
\begin{aligned}
\mathbf{U} & =\left[\begin{array}{c}
\rho \\
\rho u_{1} \\
\rho u_{2} \\
\rho u_{3} \\
\rho e
\end{array}\right], \mathbf{F}_{i}^{\text {conv }}=\rho u_{i}\left[\begin{array}{c}
1 \\
u_{1} \\
u_{2} \\
u_{3} \\
e
\end{array}\right]+p\left[\begin{array}{c}
0 \\
\delta_{1 i} \\
\delta_{2 i} \\
\delta_{3 i} \\
u_{i}
\end{array}\right], \\
\mathbf{F}_{i}^{\text {diff }} & =\left[\begin{array}{c}
0 \\
\tau_{1 i} \\
\tau_{2 i} \\
\tau_{3 i} \\
\tau_{i j} u_{j}
\end{array}\right]+\left[\begin{array}{c}
0 \\
0 \\
0 \\
0 \\
-q_{i}
\end{array}\right], \mathcal{F}=\rho\left[\begin{array}{c}
0 \\
g_{1} \\
g_{2} \\
g_{3} \\
\mathbf{g} \cdot \mathbf{u}
\end{array}\right]
\end{aligned}
$$

with $\rho=\rho(\mathbf{x}, t)$ the density and $\mathbf{u}=\mathbf{u}(\mathbf{x}, t)=\left[u_{1}, u_{2}, u_{3}\right]^{t}$ the speed which are functions of the spatial variables $\mathbf{x}=\left[x_{1}, x_{2}, x_{3}\right]^{t}$ and temporal $t ; \mathbf{g}=\left[g_{1}, g_{2}, g_{3}\right]^{t}$ the gravity, $e=\epsilon+\frac{1}{2}|\mathbf{u}|^{2}$ the total energy, sum of the internal energy $\varepsilon$ and the kinetic energy $\frac{1}{2}|\mathbf{u}|^{2}, p=p(\mathbf{x}, t)$ the pressure, $\overline{\bar{\tau}}$ the tensor of the viscous stresses, finally the quantity $\mathbf{q}(\mathbf{x}, t)=\left[q_{1}, q_{2}, q_{3}\right]^{t}$ is the thermal heat flux. In the above expressions $i, j=1,2,3$ and $\delta_{i j}$ represents the Kronecker symbol. For a Newtonian fluid, the tensor of the viscous stresses $\overline{\bar{\tau}}$ is given by:

$\overline{\bar{\tau}}=\mu\left[\nabla \mathbf{u}+(\nabla \mathbf{u})^{t}\right]+\lambda(\nabla \cdot \mathbf{u}) \overline{\bar{l}}$

with $\bar{j}$ the identity, the coefficients $\lambda$ and $\mu$ are viscosity coefficients satisfying $\lambda=-2 \mu / 3$ under the Stokes hypothesis. The thermal flux is given by the Fourier law, thus $\mathbf{q}$ and the temperature gradient $T=T(\mathbf{x}, t)$ are proportionally connected via the thermal conductivity $\kappa$ :

$\mathbf{q}=-\kappa \nabla T$

Also, compressible Navier-Stokes equations, in conservative form, are written in quasi-linear form:

$\mathbf{U}_{, t}+\mathbf{A}_{i} \mathbf{U}_{, i}=\left(\mathbf{K}_{i j} \mathbf{U}_{j}\right)_{, i}+\mathcal{F}(\mathbf{U})$

where $\mathbf{A}_{i}$ are the Jacobian matrices of the convection flows such that $\mathbf{A}_{i}=\mathbf{F}_{i, \mathbf{U}}^{\text {conv }}$ are the diffusion matrices such that $\mathbf{K}_{i j} \mathbf{U}_{j}=\mathbf{F}_{i}^{\text {diff }}$. 


\section{Unified formulation of compressible and incompressible Navier-Stokes equations}

Let the vector of the primitive variables noted $\mathrm{V}$ be given by:

$\mathbf{V}=\left[\begin{array}{c}p \\ u_{1} \\ u_{2} \\ u_{3} \\ T\end{array}\right]$

For primitive variables the quasi-linear form, compressible Navier-Stokes equations, is:

$\tilde{\mathbf{A}}_{0} \mathbf{V}_{, t}+\tilde{\mathbf{A}}_{i} \mathbf{V}_{, i}=\left(\tilde{\mathbf{K}}_{i j} \mathbf{V}_{j}\right)_{, i}+\tilde{\mathcal{F}}(\mathbf{V})$

with $\tilde{\mathbf{A}}_{0}=\mathbf{U}_{\mathbf{V}}$ is the vector transformation matrix of the conservative variables to the vector of the primitive variables, $\tilde{\mathbf{A}}_{i}=\mathbf{A}_{i} \tilde{\mathbf{A}}_{0}$ are the Jacobian matrices of the convection flows, and $\tilde{\mathbf{K}}_{i j}=\mathbf{K}_{i j} \tilde{\mathbf{A}}_{0}$ the matrices of diffusion. It should be emphasized here that matrices have a much simpler form than those derived from entropy or conservative variables, but they are neither symmetric nor positive definite, unlike those obtained with entropy variables.

To verify the incompressible limit, for the compressible Navier-Stokes equations in the quasi-linear form in primitive variables, we write the transformation matrix $\tilde{\mathbf{A}}_{0}$, and the Jacobian matrices of the convection flows $\tilde{\mathbf{A}}_{i}$, as a function of two thermodynamic parameters:

- The coefficient of thermal expansion

$$
\alpha_{p}=-\frac{1}{\rho}\left(\frac{\partial \rho}{\partial T}\right)_{p}
$$

- The coefficient of isothermal compressibility

$$
\beta_{T}=\frac{1}{\rho}\left(\frac{\partial \rho}{\partial p}\right)_{T}
$$

$$
\begin{aligned}
& \tilde{A}_{0}=\mathbf{U}_{, \mathbf{V}}=\left[\begin{array}{ccccc}
\rho \beta_{T} & 0 & 0 & 0 & -\rho \alpha_{p} \\
\rho \beta_{T} u_{1} & \rho & 0 & 0 & -\rho \alpha_{p} u_{1} \\
\rho \beta_{T} u_{2} & 0 & \rho & 0 & -\rho \alpha_{p} u_{2} \\
\rho \beta_{T} u_{3} & 0 & 0 & \rho & -\rho \alpha_{p} u_{3} \\
e_{1}^{p} & \rho u_{1} & \rho u_{2} & \rho u_{3} & e_{4}^{p}
\end{array}\right] \\
& \tilde{A}_{1}=\left[\begin{array}{ccccc}
\rho \beta_{T} u_{1} & \rho & 0 & 0 & -\rho \alpha_{p} u_{1} \\
\rho \beta_{T} u_{1}^{2}+1 & 2 \rho u_{1} & 0 & 0 & -\rho \alpha_{p} u_{1}^{2} \\
\rho \beta_{T} u_{1} u_{2} & \rho u_{2} & \rho u_{1} & 0 & -\rho \alpha_{p} u_{1} u_{2} \\
\rho \beta_{T} u_{1} u_{3} & \rho u_{3} & 0 & \rho u_{1} & -\rho \alpha_{p} u_{1} u_{3} \\
u_{1} e_{2}^{p} & \rho u_{1}^{2} & e_{3}^{p}+\rho u_{1} u_{2} & \rho u_{1} u_{3} & u_{1} e_{4}^{p}
\end{array}\right] \\
& \tilde{A}_{2}=\left[\begin{array}{ccccc}
\rho \beta_{T} u_{2} & 0 & \rho & 0 & -\rho \alpha_{p} u_{2} \\
\rho \beta_{T} u_{2} u_{1} & \rho u_{3} & \rho u_{1} & 0 & -\rho \alpha_{p} u_{2} u_{1} \\
\rho \beta_{T} u_{2}^{2}+1 & 0 & 2 \rho u_{2} & 0 & -\rho \alpha_{p} u_{2}^{2} \\
\rho \beta_{T} u_{2} u_{3} & 0 & \rho u_{3} & \rho u_{2} & -\rho \alpha_{p} u_{2} u_{3} \\
u_{2} e_{2}^{p} & \rho u_{2} u_{1} & e_{3}^{p}+\rho u_{2}^{2} & \rho u_{2} u_{3} & u_{2} e_{4}^{p}
\end{array}\right] \\
& \tilde{A}_{3}=\left[\begin{array}{ccccc}
\rho \beta_{T} u_{3} & 0 & 0 & \rho & -\rho \alpha_{p} u_{3} \\
\rho \beta_{T} u_{3} u_{1} & \rho u_{3} & 0 & \rho u_{1} & -\rho \alpha_{p} u_{3} u_{1} \\
\rho \beta_{T} u_{3} u_{2} & 0 & \rho u_{3} & \rho u_{2} & -\rho \alpha_{p} u_{3} u_{2} \\
\rho \beta_{T} u_{3}^{2}+1 & 0 & 0 & 2 \rho u_{3} & -\rho \alpha_{p} u_{3}^{2} \\
u_{3} e_{2}^{p} & \rho u_{3} u_{1} & \rho u_{3} u_{2} & e_{3}^{p}+\rho u_{3}^{2} & u_{3} e_{4}^{p}
\end{array}\right]
\end{aligned}
$$

The matrix matrices of diffusion, are given by:

$$
\begin{aligned}
\tilde{K}_{11} & =\left[\begin{array}{ccccc}
0 & 0 & 0 & 0 & 0 \\
0 & \chi & 0 & 0 & 0 \\
0 & 0 & \mu & 0 & 0 \\
0 & 0 & 0 & \mu & 0 \\
0 & \chi u_{1} & \mu u_{2} & \mu u_{3} & \kappa
\end{array}\right] \\
\tilde{K}_{12} & =\left[\begin{array}{ccccc}
0 & 0 & 0 & 0 & 0 \\
0 & 0 & \lambda & 0 & 0 \\
0 & \mu & 0 & 0 & 0 \\
0 & 0 & 0 & 0 & 0 \\
0 & \mu u_{2} & \lambda u_{1} & 0 & 0
\end{array}\right] \\
\tilde{K}_{13} & =\left[\begin{array}{ccccc}
0 & 0 & 0 & 0 & 0 \\
0 & 0 & 0 & \lambda & 0 \\
0 & 0 & 0 & 0 & 0 \\
0 & \mu & 0 & \mu & 0 \\
0 & \mu u_{3} & 0 & \lambda u_{1} & 0
\end{array}\right]
\end{aligned}
$$


$\begin{aligned} \tilde{K}_{21} & =\left[\begin{array}{ccccc}0 & 0 & 0 & 0 & 0 \\ 0 & 0 & \mu & 0 & 0 \\ 0 & \lambda & 0 & 0 & 0 \\ 0 & 0 & 0 & 0 & 0 \\ 0 & \lambda u_{2} & \mu u_{1} & 0 & 0\end{array}\right] \\ \tilde{K}_{22}= & {\left[\begin{array}{ccccc}0 & 0 & 0 & 0 & 0 \\ 0 & \mu & 0 & 0 & 0 \\ 0 & 0 & \chi & 0 & 0 \\ 0 & 0 & 0 & \mu & 0 \\ 0 & \mu u_{1} & \chi u_{2} & \mu u_{3} & \kappa\end{array}\right] }\end{aligned}$

$\tilde{K}_{23}=\left[\begin{array}{ccccc}0 & 0 & 0 & 0 & 0 \\ 0 & 0 & 0 & 0 & 0 \\ 0 & 0 & 0 & \lambda & 0 \\ 0 & 0 & \mu & 0 & 0 \\ 0 & 0 & \mu u_{3} & \lambda u_{2} & 0\end{array}\right]$

$\tilde{K}_{31}=\left[\begin{array}{ccccc}0 & 0 & 0 & 0 & 0 \\ 0 & 0 & 0 & \mu & 0 \\ 0 & 0 & 0 & 0 & 0 \\ 0 & \lambda & 0 & 0 & 0 \\ 0 & \lambda u_{3} & 0 & \mu u_{1} & 0\end{array}\right]$

$\tilde{K}_{32}=\left[\begin{array}{ccccc}0 & 0 & 0 & 0 & 0 \\ 0 & 0 & 0 & 0 & 0 \\ 0 & 0 & 0 & \mu & 0 \\ 0 & 0 & \lambda & 0 & 0 \\ 0 & 0 & \lambda u_{3} & \mu u_{2} & 0\end{array}\right]$

$\tilde{K}_{33}=\left[\begin{array}{ccccc}0 & 0 & 0 & 0 & 0 \\ 0 & \mu & 0 & 0 & 0 \\ 0 & 0 & \mu & 0 & 0 \\ 0 & 0 & 0 & \chi & 0 \\ 0 & \mu u_{1} & \mu u_{2} & \chi u_{3} & \kappa\end{array}\right]$

or:

$e_{1}^{p}=\rho \beta_{T} e_{1}-\alpha_{p} T, e_{2}^{p}=e_{1}^{p}+1, e_{3}^{p}=\rho e_{1}, e_{4}^{p}=-\rho \alpha_{p} e_{1}+\rho c_{p}$

$e_{1}=h+\frac{|\mathbf{u}|^{2}}{2}$

$\chi=\lambda+2 \mu$

The incompressible limit of compressible Navier-Stokes equations, quasi-linear (3), is obtained when the coefficients of thermal expansion $\alpha_{p}$ and of isothermal compressibility $\beta_{T}$ are taken equal to zero and when the density $\rho$ is supposed constant:

$\alpha_{p}=0 \quad \beta_{T}=0$ and $\rho=c t e$

Using the properties (6), fluid with zero compressibility $\left(\beta_{T}=0\right)$ and zero expansion $\left(\alpha_{p}=0\right)$, in the Jacobian matrices written above, we obtain:

$$
\begin{aligned}
\tilde{A}_{0}^{i n c}= & {\left[\begin{array}{lllll}
0 & 0 & 0 & 0 & 0 \\
0 & \rho & 0 & 0 & 0 \\
0 & 0 & \rho & 0 & 0 \\
0 & 0 & 0 & \rho & 0 \\
0 & \rho u_{1} & \rho u_{2} & \rho u_{3} & \rho c_{p}
\end{array}\right] } \\
\tilde{A}_{1}^{i n c}= & {\left[\begin{array}{ccccc}
0 & \rho & 0 & 0 & 0 \\
1 & 2 \rho u_{1} & 0 & 0 & 0 \\
0 & \rho u_{2} & \rho u_{1} & 0 & 0 \\
0 & \rho u_{3} & 0 & \rho u_{1} & 0 \\
u_{1} & \rho e_{1}+\rho u_{1}^{2} & \rho u_{1} u_{2} & \rho u_{1} u_{3} & u_{1} \rho c_{p}
\end{array}\right] } \\
\tilde{A}_{2}^{i n c}= & {\left[\begin{array}{ccccc}
0 & 0 & \rho & 0 & 0 \\
0 & \rho u_{2} & \rho u_{1} & 0 & 0 \\
1 & 0 & 2 \rho u_{2} & 0 & 0 \\
0 & 0 & \rho u_{3} & \rho u_{2} & 0 \\
u_{2} & \rho u_{2} u_{1} & \rho e_{1}+\rho u_{2}^{2} & \rho u_{2} u_{3} & u_{2} \rho c_{p}
\end{array}\right] } \\
\tilde{A}_{3}^{i n c}= & {\left[\begin{array}{ccccc}
0 & 0 & 0 & \rho & 0 \\
0 & \rho u_{3} & 0 & \rho u_{1} & 0 \\
0 & 0 & \rho u_{3} & \rho u_{2} & 0 \\
1 & 0 & 0 & 2 \rho u_{3}^{2} & 0 \\
u_{3} & \rho u_{3} u_{1} & \rho u_{3} u_{2} & \rho e_{1}+\rho u_{3}^{2} & u_{3} \rho c_{p}
\end{array}\right] }
\end{aligned}
$$

The matrices of the diffusion flows, as for them are unchanged because they do not depend on $\alpha_{p}$ nor of $\beta_{T}$. We conclude that, for the primitive variables, it is possible to write in a unified way the compressible and incompressible quasi-linear equations.

\section{Stabilized finite element method}

The resolution of the Navier-Stokes equations is done using a stabilized finite element method of the Petrov-Galerkin type.

\subsection{Galerkin formulation}

Let a time interval $I=10, T$ [ discretized into $n$ segments $\left.I_{n}=\right] t_{n}, t_{n+1}[$; and a domain $\Omega$ of border $\Gamma$. Time-discretized domains are also noted

$\Omega_{n}=\Omega \times I_{n}$ and $\Gamma_{n}=\Gamma \times I_{n}$

For spatial discretization, the domain $\Omega_{n}$ is subdivided into $\left(n_{e l}\right)_{n}$ elements making it possible to define the discretization domain on each element

$\Omega_{n}^{e}=\left[\Omega \times I_{n}\right]^{e}, \quad e \in\left[1, \ldots,\left(n_{e l}\right)_{n}\right]$

The interpolation $\Phi$ and weighting $\mathbf{W}$ functions are taken in the following definition domains: $\Phi \in \phi_{\mathrm{n}}$ and $\mathbf{W} \in \mathrm{w}_{\mathrm{n}}$. Either the equation system to solve (3) 
$\tilde{\mathbf{A}}_{0} \mathbf{V}_{, t}+\tilde{\mathbf{A}}_{i} \mathbf{V}_{, i}=\left(\tilde{\mathbf{K}}_{i j} \mathbf{V}_{j}\right)_{, i}+\tilde{\mathcal{F}}(\mathbf{V})$

The variational formulation of the problem is then expressed in the form: find $\mathbf{V} \in \phi_{\mathrm{n}}$ such that for all $\mathbf{W} \in \mathrm{w}_{\mathrm{n}}$

$\int_{\Omega_{n}} \mathbf{W} \cdot\left(\tilde{\mathbf{A}}_{0} \mathbf{V}_{, t}+\tilde{\mathbf{A}}_{i} \mathbf{V}_{, i}-\left(\tilde{\mathbf{K}}_{i j} \mathbf{V}_{j}\right)_{, i}-\tilde{\mathcal{F}}\right) \mathrm{d} \Omega_{n}=0$

is

$\int_{\Omega_{n}} \mathbf{W} \cdot\left(\tilde{\mathbf{A}}_{0} \mathbf{V}_{, t}+\mathcal{L}(\mathbf{V})-\tilde{\mathcal{F}}\right) \mathrm{d} \Omega_{n}=0$

where the stationary Navier-Stokes $\mathcal{L}$ operator is defined by

$$
\mathcal{L}=\tilde{\mathbf{A}}_{i} \frac{\partial}{\partial x_{i}}-\frac{\partial}{\partial x_{i}}\left(\tilde{\mathbf{K}}_{i j} \frac{\partial}{\partial x_{j}}\right)
$$

The weak formulation associated with the variational problem consists of integrating the flows of Eq. (7) into parts. We therefore seek to find $\mathbf{V} \in \phi_{\mathrm{n}}$ such that for all $\mathbf{W} \in \mathrm{w}_{n}$

$$
\begin{aligned}
& \int_{\Omega_{n}} \mathbf{W} \cdot\left(\tilde{\mathbf{A}}_{0} \mathbf{V}_{, t}+\tilde{\mathbf{A}}_{i} \mathbf{V}_{, i}-\tilde{\mathcal{F}}\right)+\mathbf{W}_{, i} \cdot\left(\tilde{\mathbf{K}}_{i j} \mathbf{V}_{j}\right) \mathrm{d} \Omega_{n} \\
& =\oint_{\Gamma_{n}} \mathbf{W} \cdot\left[\left(\tilde{\mathbf{K}}_{i j} \mathbf{V}_{j}\right) \cdot \mathbf{n}_{i}\right] \mathrm{d} \Gamma_{n}
\end{aligned}
$$

Equation (9) is the Galerkin space-time formulation. The first integral represents the volume part of the integration by part of Eq. (7). The second integral represents the boundary of integration by part of the stationary term.

\subsection{Petrov-Galerkin formulation}

The Petrov-Galerkin formulation consists of stabilizing the Galerkin formulation by replacing the weighting function term $\mathbf{W}$ with $\mathbf{W}+\mathbf{P}(\mathbf{W})$ where $\mathbf{P}$ is a perturbation function of $\mathbf{W}$. There are different methods for modeling the perturbation $\mathbf{P}(\mathbf{W})$.

The Streamline Upwind Petrov-Galerkin (SUPG) method is based on the concept of upstream decentering and is equivalent to artificial diffusivity methods. In the context of the incompressible Navier-Stokes equations, [1] propose the following generalized definition to the compressible case in entropy variables

$\mathbf{P}(\mathbf{W})=\tau \tilde{A}_{i} \frac{\partial \mathbf{W}}{\partial x_{i}}$

The matrix $\tau$ is called the stabilization matrix.
The Galerkin Least Square (GLS) method is based on a linear combination of the diffusive and convective term. Typically, it is written:

$\mathbf{P}(\mathbf{W})=\tau \mathcal{L}(\mathbf{W})$

Thus the weak formulation Galerkin (9) is rewritten with the additional term

$$
\begin{gathered}
\int_{\Omega_{n}} \mathbf{W} \cdot\left(\tilde{\mathbf{A}}_{0} \mathbf{V}_{, t}+\tilde{\mathbf{A}}_{i} \mathbf{V}_{, i}-\tilde{\mathcal{F}}\right)+\mathbf{W}_{, i} \cdot\left(\tilde{\mathbf{K}}_{i j} \mathbf{V}_{j}\right) \mathrm{d} \Omega_{n} \\
+\sum_{e} \int_{\Omega^{e}} \mathcal{L}(\mathbf{W}) \cdot \tau\left(\tilde{\mathbf{A}}_{0} \mathbf{V}_{, t}+\mathcal{L}(\mathbf{V})-\tilde{\mathcal{F}}\right) \mathrm{d} \Omega^{e} \\
=\oint_{\Gamma_{n}} \mathbf{W} \cdot\left[\left(\tilde{\mathbf{K}}_{i j} \mathbf{V}_{j}\right) \cdot \mathbf{n}_{i}\right] \mathrm{d} \Gamma_{n}
\end{gathered}
$$

The consistency of the stabilization term is ensured since it is multiplied by the residue. It tends to zero when the solution converges. It should also be noted that the stabilization matrix $\tau$ is defined only on the element, hence the presence of the term sum around the local integral. There is no nodal definition of stabilization. Thus the stabilization term has no global meaning as a physical field.

\section{Results and discussions}

Supersonic flows, as well as those in the transonic regime, are characterized by the presence of shock waves, which is a major challenge for numerical resolution, because of the strong gradients that are present (physically, we even talk about of a discontinuity). The nature of the flow, especially for the transonic flow, also complicates resolution by numerical methods. Mathematically, it is shown that the partial differential equations, describing the flows, vary from an elliptic nature under a subsonic regime to a hyperbolic nature under a supersonic regime. The properties of the solutions are fundamentally different; the parabolic equations generally describe phenomena of dissipation or diffusion, whereas the hyperbolic equations characterize the propagation phenomena of waves or vibrations. It is therefore difficult to define a resolution algorithm favoring both regimes. For flows with high Reynolds numbers, the shock that appears during a supersonic regime compression at the subsonic regime is comparable to a zone of strong gradients. It is difficult, if not impossible, to predict for any flow the location of the shock, governed by the counterpressure. A strong impact can cause the boundary layer to detach, thus changing the pressure distribution downstream. In order to interpret the results obtained using the techniques mentioned in the preceding paragraphs, we will discuss, in this section, the physical 
phenomena that characterize transonic and supersonic flows: supersonic zone and shock wave.

\subsection{Non-viscous compressible flow: shock reflection}

We consider a non-viscous, compressible and two-dimensional flow; this flow is governed by the Euler equations. The interest of dealing with this problem lies in the simulation of flows where oblique shock waves are present. The flow comprises three different regions, separated by an oblique shock and its reflection on the wall. The exact solution includes an incident shock wave, directed at $29^{\circ}$ from the horizontal, which reflects off the wall at an angle of $23^{\circ}$. The computational domain is a rectangle of length 4.1 on the $x$-axis and width 1.0 on the $y$-axis.

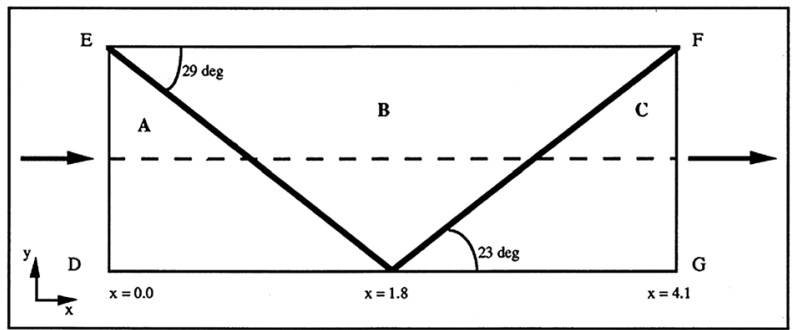

The exact solution of the flow in the three regions is given by: region $\mathrm{A}$

$\left\{\begin{array}{l}M_{A}=2.9 \\ \rho_{A}=1.0 \\ \rho u_{1_{A}}=2.9 \\ \rho u_{2_{A}}=0.0 \\ p_{A}=0.71143 \\ \rho e_{A}=5.9907\end{array}\right.$

region $B$

$$
\left\{\begin{array}{l}
M_{B}=2.3781 \\
\rho_{B}=1.7 \\
\rho u_{1_{B}}=4.452868 \\
\rho u_{2_{B}}=-0.86071 \\
p_{B}=1.52819 \\
\rho e_{B}=9.870138
\end{array}\right.
$$

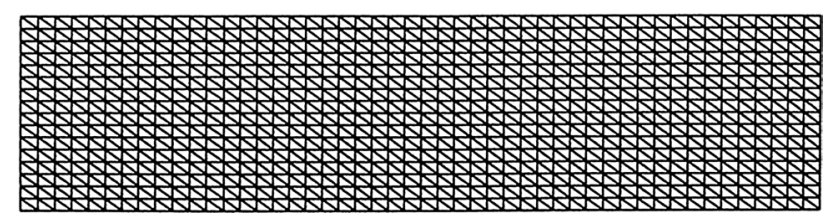

Fig. 1 Shock reflection problem-first mesh in region $\mathrm{C}$, the oblique shock wave theory shows that we have:

$\left\{\begin{array}{l}M_{C}=1.942 \\ \rho_{C}=2.6872838 \\ \rho u_{1_{C}}=4.664552 \\ \rho u_{2_{C}}=0.0 \\ p_{C}=2.93398\end{array}\right.$

The numerical solution is obtained on two meshes. The first is a structured mesh composed of 1536 triangular elements and 3201 nodes, illustrated in Fig. 1. The second is an unstructured mesh, obtained after refinement and derefinement of the first mesh, composed of 4387 triangular elements and 8870 nodes, illustrated in Fig. 2.

For the boundary conditions, at the entry of the flow, boundary [DE[, all the variables are fixed at the values of the exact solution of the region $A$. While on the upper boundary $[E F[$, the variables are fixed at the values of the exact solution of the region B. On the lower boundary ]DG], or imposes the sliding condition of the fluid on the wall: $\mathbf{u} \cdot \mathbf{n}=0$. At the exit of the flow, boundary ]GF], all the variables are left free. The primary objective is to demonstrate that the oscillations in the vicinity of the shock are controllable. For this, we solve this problem on the first mesh, the evolution in time is carried out with local time steps, $C F L=0.7$. In Figs. 3, 4, 5 and 6, the Iso-pressure lines and the pressure section are presented at $y=0.7$.

From these results, it is clear that the oscillations are well controlled and that they are almost eliminated. Also, these results show that a better shock resolution is obtained when the mesh is adapted. On the second mesh, the resolution of incident and reflected shocks is better than that on the first mesh. The shock is better captured and the oscillations in its vicinity have completely disappeared compared to other formulations. This shows that

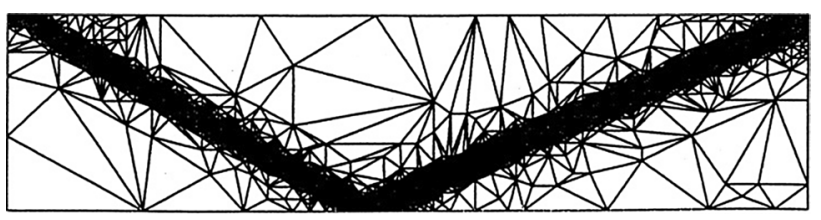

Fig. 2 Shock reflection problem—second mesh

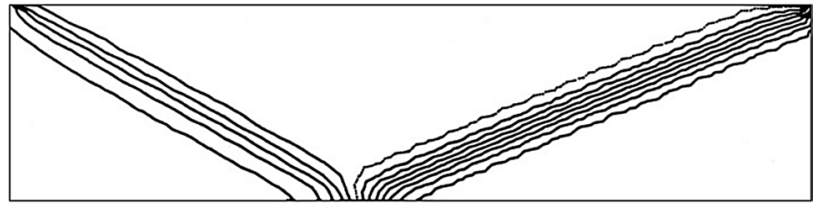

Fig. 3 Iso-pressure lines, viscous compressible flow-first mesh 


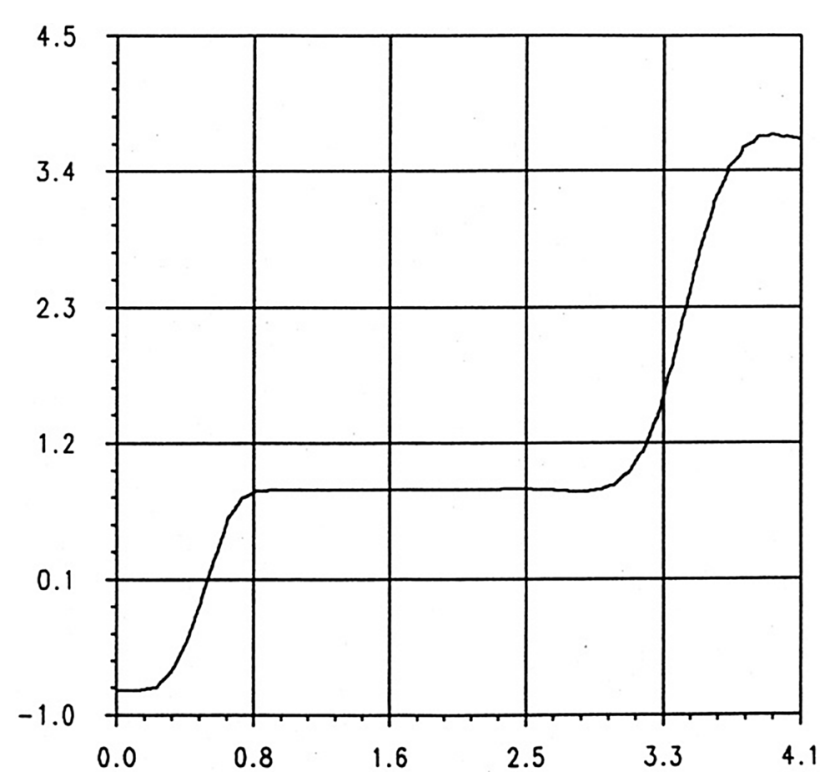

Fig. 4 Pressure cut at $y=0.7$, viscous compressible flow-first mesh

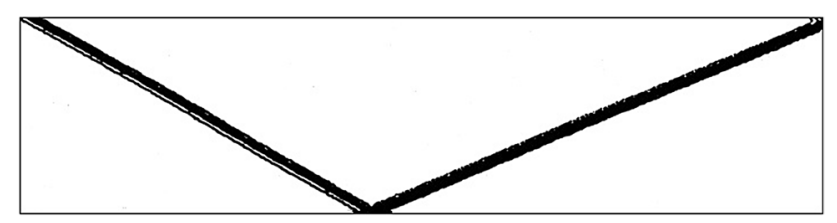

Fig. 5 Iso-pressure lines, viscous compressible flow—second mesh

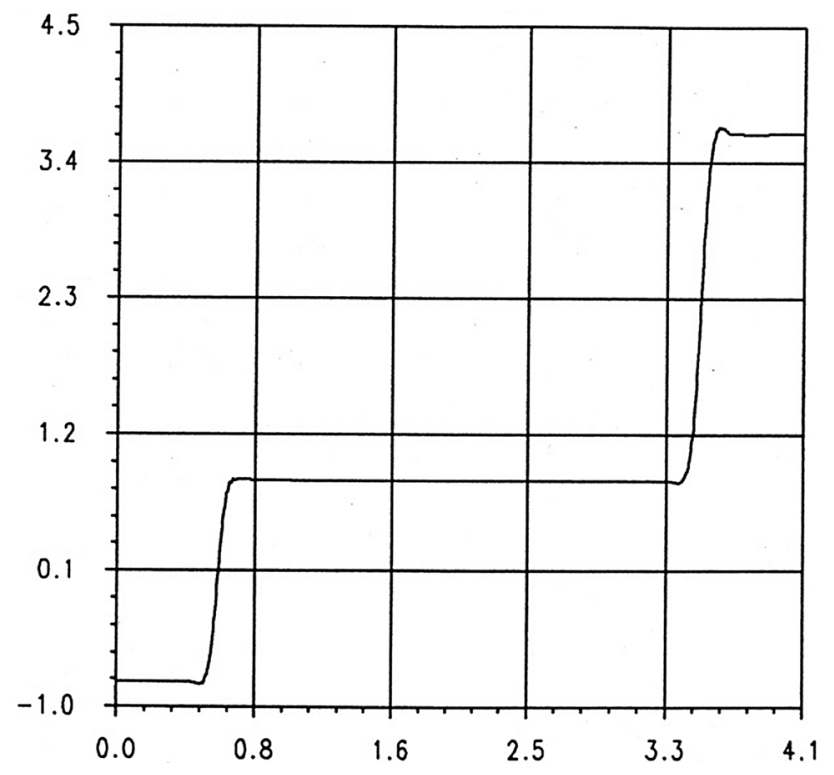

Fig. 6 Pressure section at $y=0.7$, viscous compressible flow-second mesh this formulation captures shocks well and does not disturb the solution in the smooth regions.

\subsection{Viscous compressible flow: flow on a flat plate}

This case test shows a supersonic flow at Mach 3, for a viscous fluid along a flat plate. The computational domain is a rectangle of length 1.6 on the $x$-axis and width 1.0 on the y-axis.

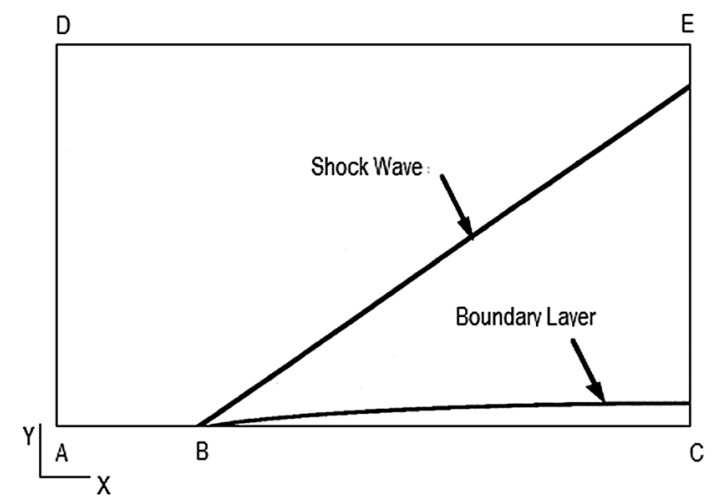

The numerical solution is obtained on a structured mesh, composed of 2560 triangular elements and 5265 nodes. For the boundary conditions, at the inlet of the flow $[A D]$ and at the upper boundary $[D E]$, all the variables are fixed at the values at infinity:

$\left\{\begin{array}{l}M_{\infty}=3.0 \\ \rho=\rho_{\infty} \\ \rho u_{1}=U_{\infty} \\ \rho u_{1}=0.0 \\ \rho e=E_{\infty}\end{array}\right.$

At the exit of the flow, border ]EC [, all the variables are left free. On the boundary $] A B$ [, one imposes the horizontal component of the speed is equal to zero. On the plane plate, border $[B C]$, one imposes the condition of adhesion $\mathbf{u}=0$ and the condition through the temperature:

$T=T_{\text {stag }}=T_{\infty}\left(1+\frac{\gamma-1}{2} M_{\infty}^{2}\right)$

The initial solution used is a uniform field, except on the plate $[B C]$ where the normal and tangential component of the velocity is imposed equal to zero. The resolution strategy consists of setting the Reynolds number to 1000 and then to 10,000 and the Mach number to 3, the step in time is carried out with local time steps, $C F L=0.5$. Under these physical conditions, there is a boundary layer and an oblique shock. For the Reynolds number is equal to 1000 , the Iso-density, Iso-Mach and Iso-pressure lines are shown in Figs. 7,8 and 9. 


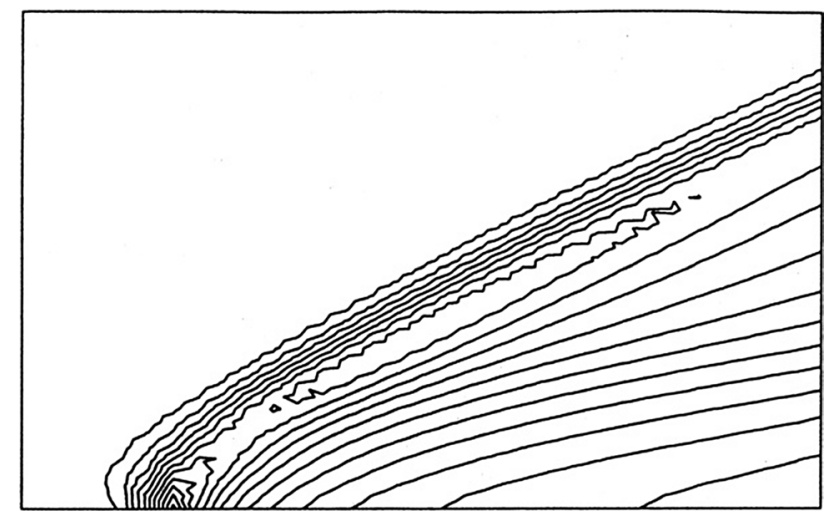

Fig. 7 Iso-density lines, viscous flow on a flat plate-Ma=3.0 and $\operatorname{Re}=1000$

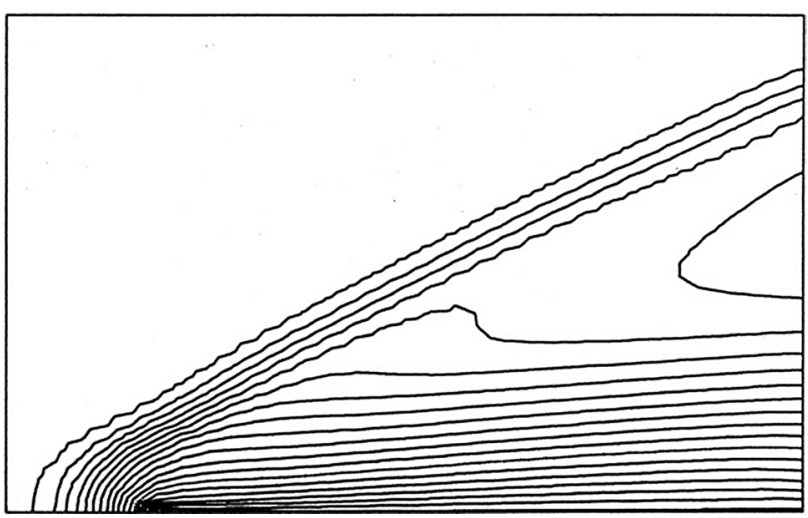

Fig. 8 Iso-Mach line, viscous flow on a flat plate-Ma=3.0 and $\operatorname{Re}=1000$

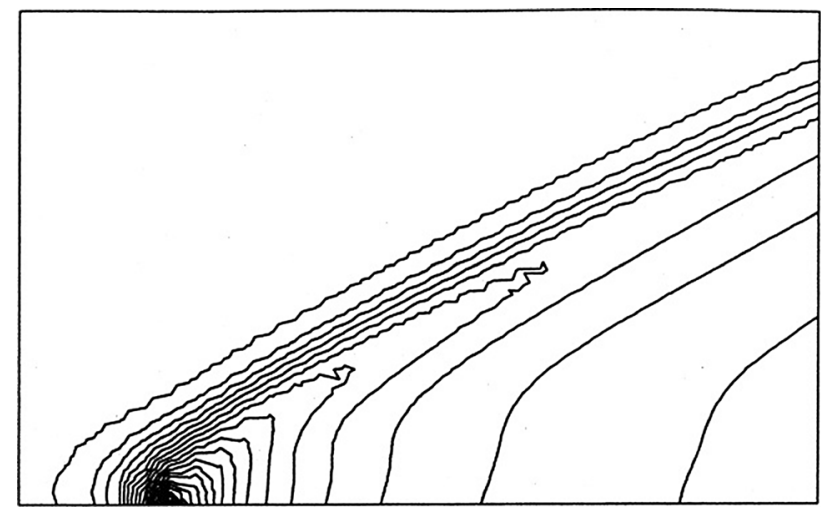

Fig. 9 Iso-pressure line, viscous flow on a flat plate-Ma=3.0 and $R e=1000$

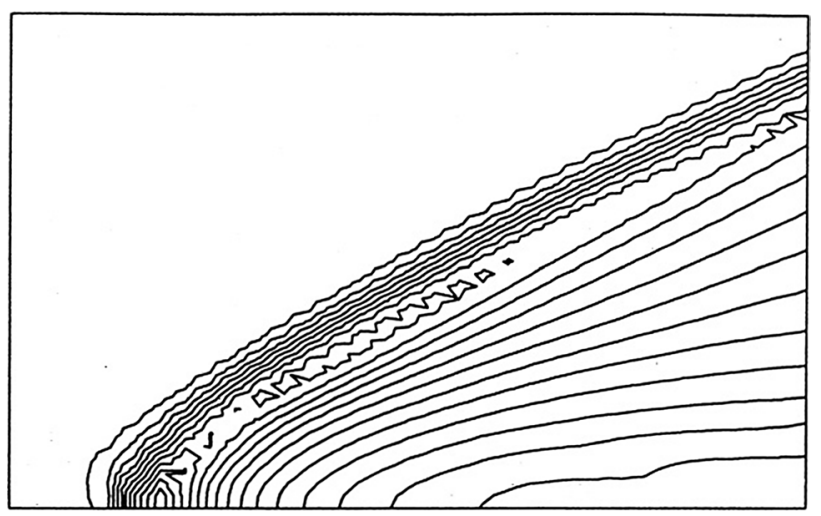

Fig. 10 Iso-density lines, viscous flow on a flat plate-Ma=3.0 and $R e=10,000$

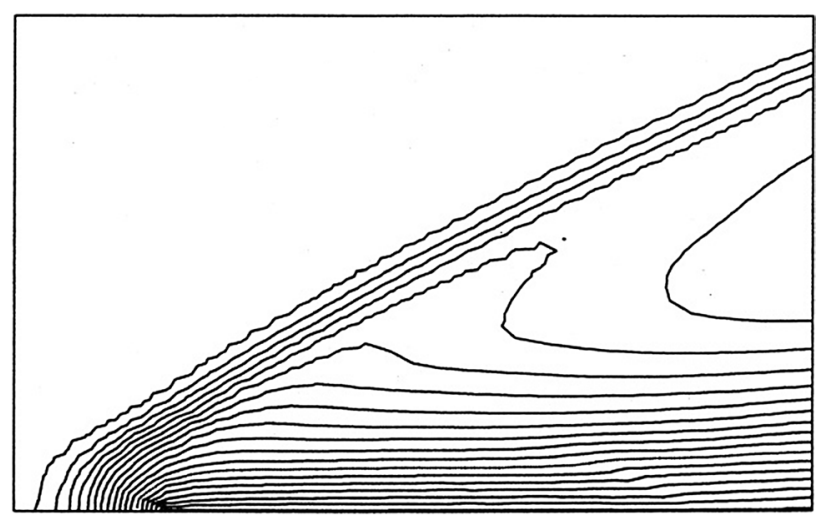

Fig. 11 Iso-Mach lines, viscous flow on a flat plate-Ma=3.0 and $R e=10,000$

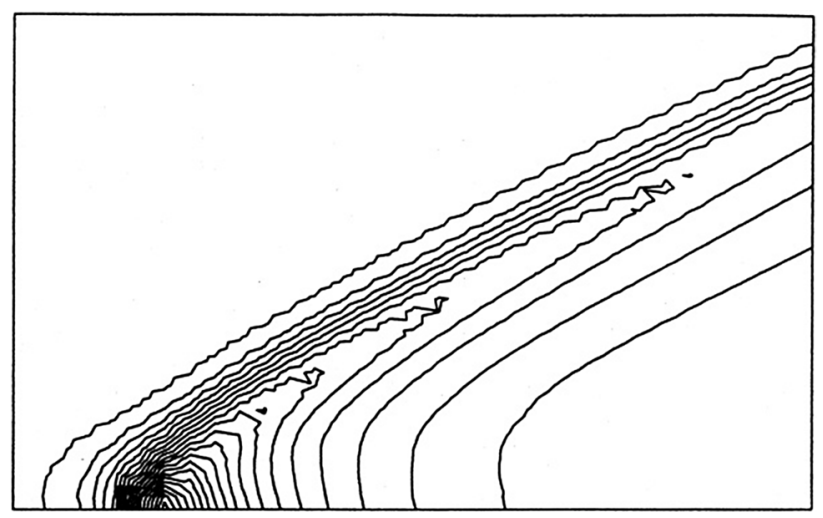

Fig. 12 Iso-pressure lines, viscous flow on a flat plate $-M a=3.0$ and $\operatorname{Re}=10,000$ 


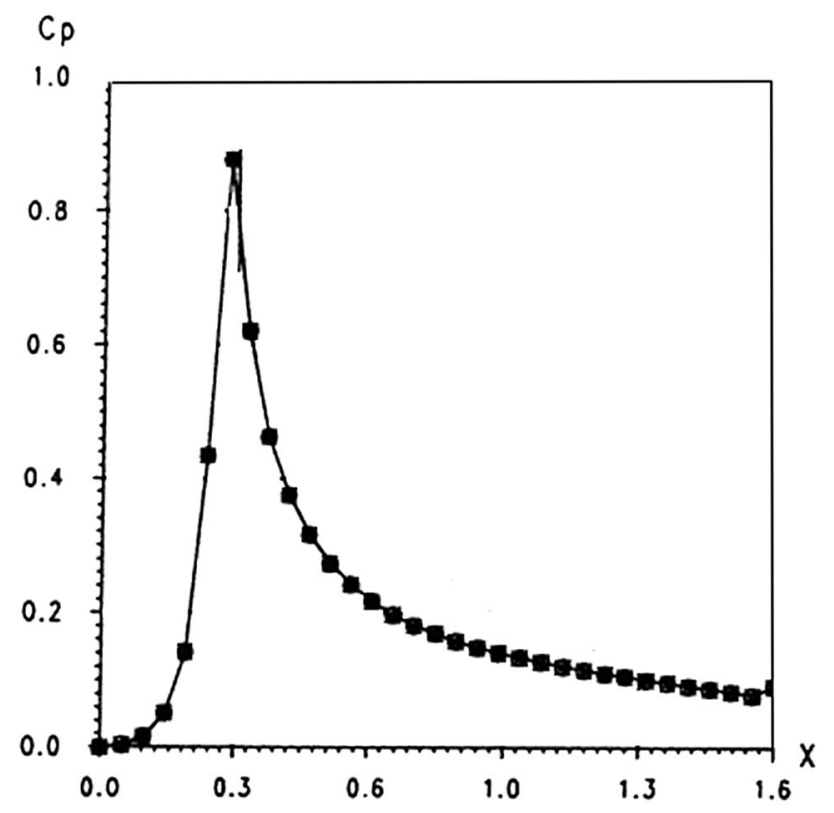

Fig. 13 Pressure distribution, viscous flow on a flat plate-Ma=3.0 and $R e=1000$

For the Reynolds number is equal to 10,000 the Iso-density, Iso-Mach and Iso-pressure lines are shown in Figs. 10, 11 and 12.

The pressure coefficients are compared with those obtained by a finite element method, using the entropy variables (Shakib et al. [32]), Fig. 13 for a Reynolds number of 1000, Fig. 14 for a Reynolds number of 10,000.

\subsection{Viscous incompressible flow: cavity problem}

The cavity problem has become a classic test case, to evaluate the accuracy and efficiency of numerical methods used for the resolution of incompressible flows in laminar flow. The computational domain is a rectangle of length 1.0 on the $x$-axis and width 1.0 on the $y$-axis.

The numerical solution is obtained on a structured mesh, composed of 800 triangular elements and 1681 nodes, illustrated on Fig. 15.

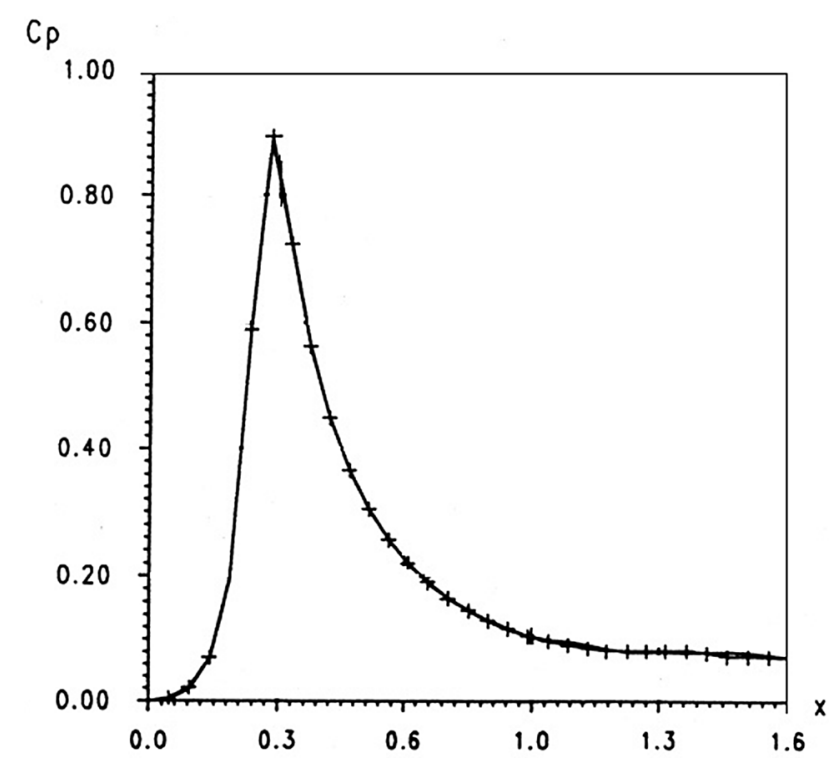

Fig. 14 Pressure distribution, viscous flow on a flat plate-Ma=3.0 and $R e=10,000$

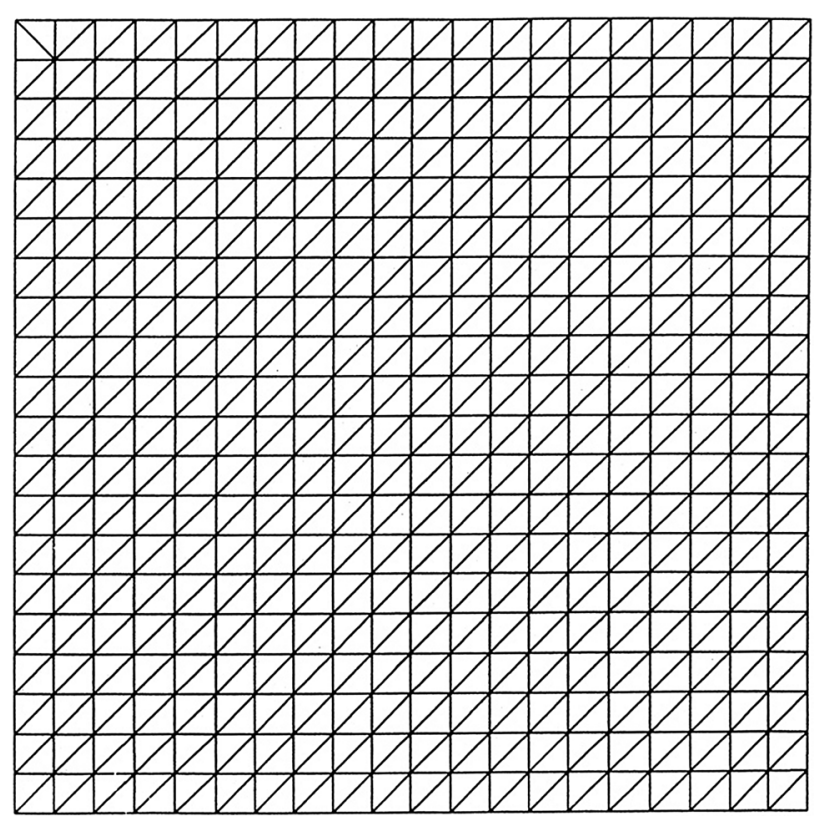

Fig. 15 Problem of the cavity-mesh 


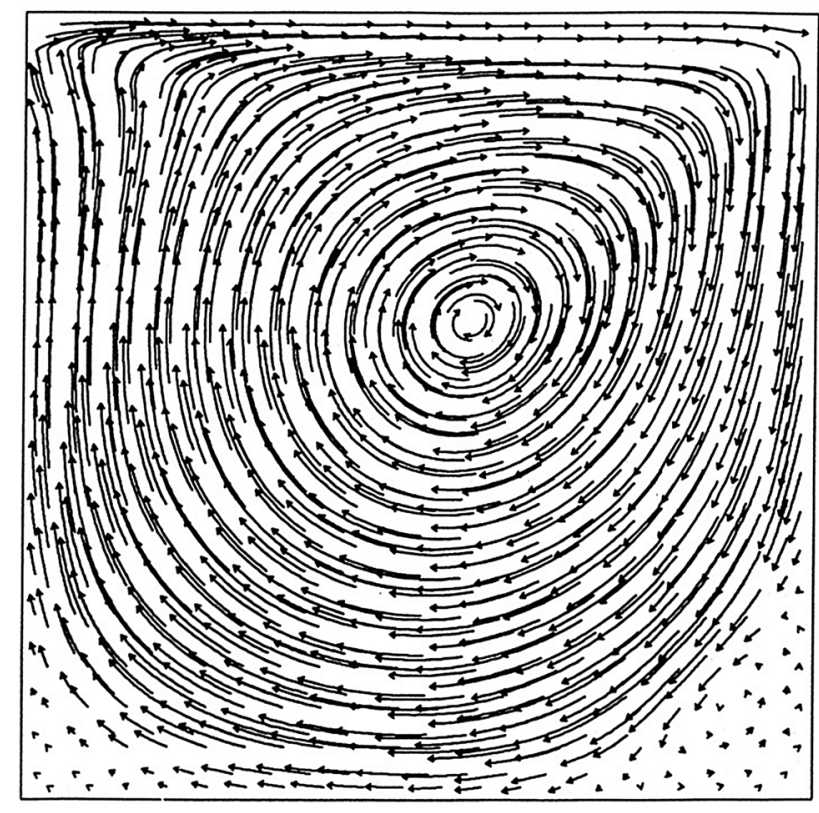

Fig. 16 Velocity fields, viscous incompressible flow $R e=400$

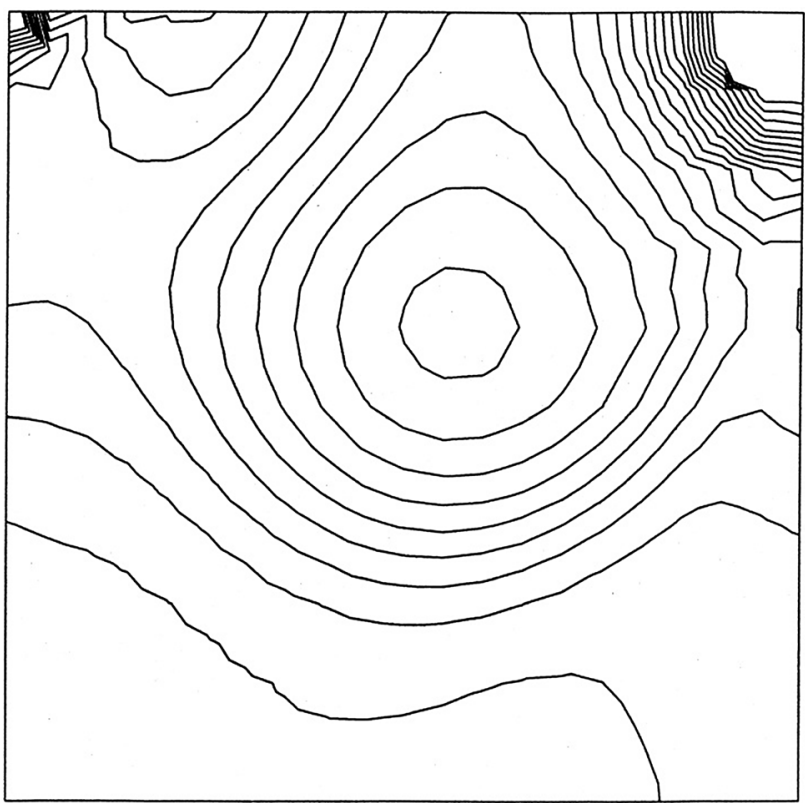

Fig. 17 Iso-pressure lines, viscous incompressible flow $R e=400$

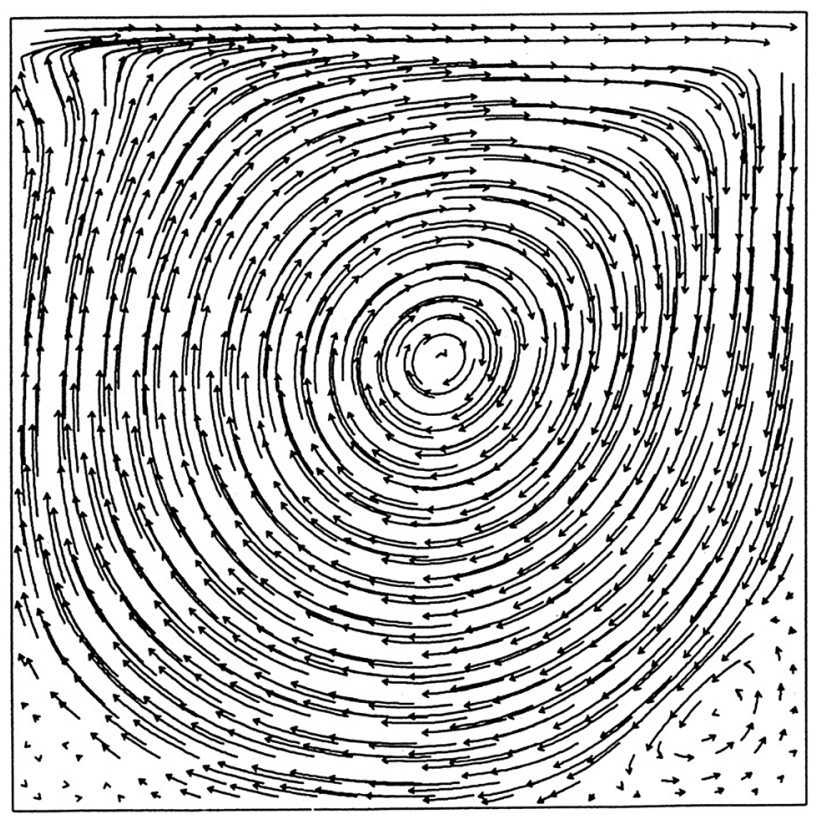

Fig. 18 Velocity fields, viscous incompressible flow $R e=1000$

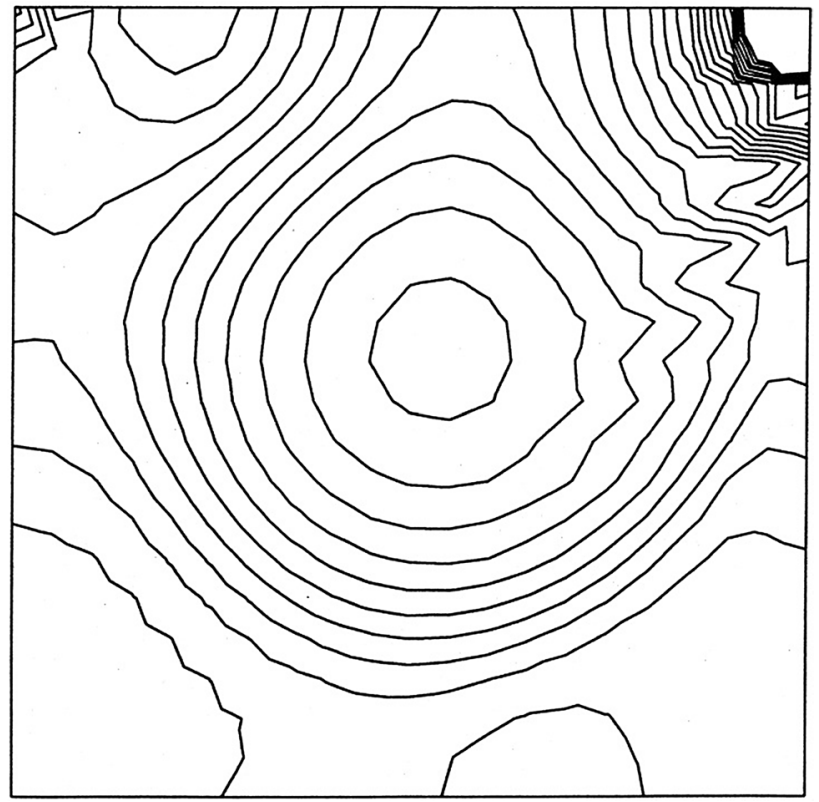

Fig. 19 Iso-pressure lines, viscous incompressible flow $R e=1000$ 


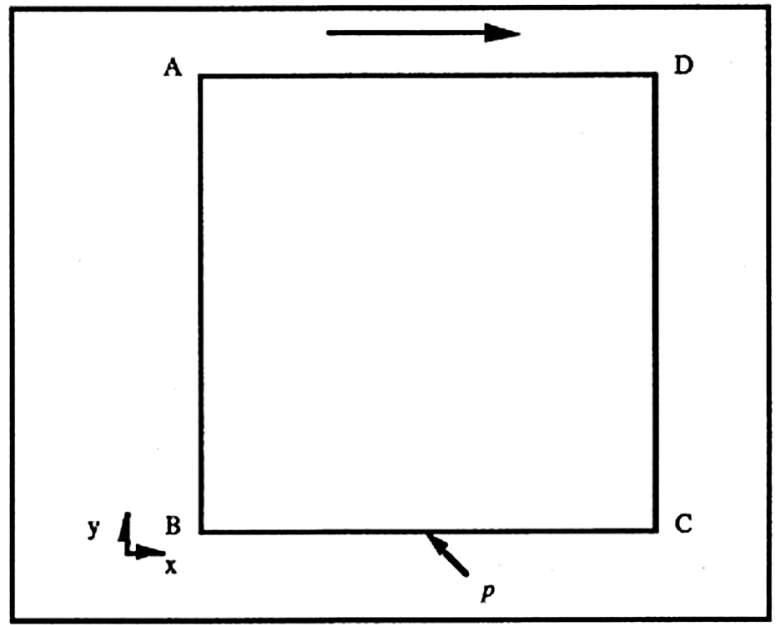

For boundary conditions, on the upper boundary $] A D[$

$\left\{\begin{array}{l}u_{1}=1.0 \\ u_{2}=0.0\end{array}\right.$

on Borders $[A B],[B C]$ and $[C D$

$\left\{\begin{array}{l}u_{1}=0.0 \\ u_{2}=0.0\end{array}\right.$

The pressure $p$ is set to a value for a node of the boundary $[B C]$. For flow at $R e=400$, the velocity field is shown in Fig. 16 and the Iso-pressure lines in Fig. 17.

For the flow at $R e=1000$, the velocity field in Fig. 18 and the Iso-pressure lines in Fig. 19 are presented.

These results have been compared with those of Deschênes [33], Hauke and Hughes [25] and Franca and Frey [3] obtained by a finite element method. As noted by $[3,25,33]$, there is a large central vortex accompanied by smaller vortices of reverse rotation in the lower corners of the cavity. The results obtained are satisfactory and comparable to the results of the previous work, hence the validation of the method developed.

\section{Conclusion and perspectives}

We have presented different formulations of compressible and incompressible Navier-Stokes equations. In particular, we have emphasized the existence of a unified, quasilinear formulation for primitive variables. This means that a compressible or incompressible flow can be described by this unified formulation of Navier-Stokes equations using adequate matrices. This unified formulation is parameterized by the density and two thermodynamic coefficients: thermal expansion and isothermal compressibility, which are strongly related to the nature of the fluid considered. For an incompressible fluid the density is supposed constant and $\alpha_{p}$ and $\beta_{T}$ are zero. While for a compressible fluid these quantities are strongly related to the state law used.

The numerical method considered in this work is of the finite element type. When Lagrange polynomials of the same degree are used to approach each unknown (pressure, velocity and temperature for example), the Galerkin formulation applied to compressible and incompressible Navier-Stokes equations presents numerical instabilities which it is necessary to stabilize. For this we introduce a disturbance weighted by a stabilization parameter to ensure the accuracy and consistency of the numerical scheme. It is in this context that Petrov-Galerkin formulations have been introduced: SUPG or GLS. Here, we will retain the last method which allows obtaining a valid unified numerical scheme for the Navier-Stokes equations, compressible and incompressible flows.

The corresponding software, in its current version, is only dedicated to solving $2 \mathrm{D}$ problems. Its extension in $3 D$ is envisaged and can be done in a rather natural way.

\section{Compliance with ethical standards}

Conflict of interest The authors declare that they have no conflict of interest. 


\section{References}

1. Brooks AN, Hughes TJR (1982) Streamline upwind/Petrov-Galerkin formulations for convection dominated flows with particular emphasis on the incompressible Navier-Stokes equations. Comput Methods Appl Mech Eng 32:199-259

2. Hughes TJR, Franca LP, Balestra M (1986) A new finite element formulation for computational fluid dynamics: V. Circumventing the babuska-brezzi condition: a stable Petrov-Galerkin formulation for the stokes problem accommodating equal order interpolations. Comput Methods Appl Mech Eng 59:85-99

3. Franca LP, Frey SL (1992) Stabilized finite element methods: II. The incompressible Navier-Stokes equations. Comput Methods Appl Mech Eng 99:209-233

4. Shakib F, Hughes TJR (1991) A new finite element formulation for computational fluid dynamics: X. The compressible Euler and Navier-Stokes equations. Comput Methods Appl Mech Eng 89:141-219

5. Brezzi F, Bristeau M-O, Franca LP, Mallet M, Roge G (1991) A relationship between stabilized finite element methods and the Galerkin method with bubble functions. Comput Methods Appl Mech Eng 96(1):117-129

6. Hughes TJR (1995) Multiscale phenomena: Green's functions, the Dirichlet-to-Neumann formulation subgrid scale models, bubbles and the origins of stabilized methods. Comput Methods Appl Mech Eng 127(1-4):387-401

7. Masud A, Khurram RA (2006) A multiscale finite element method for the incompressible Navier-Stokes equations. Comput Methods Appl Mech Eng 195:1750-1777

8. Codina R (2002) Stabilized finite element approximation of transient incompressible flows using orthogonal subscales. Comput Methods Appl Mech Eng 191(39-40):4295-4321

9. Burman E, Ern A (2007) Continuous interior penalty hp-finite element methods for advection and advection-diffusion equations. Math Comput 76(259):1119-1141

10. Hughes TJR, Franca LP, Hulbert GM (1989) A new finite element formulation for computational fluid dynamics: VIII. The Galerkin/ least-squares method for advective-diffusive equations. Comput Methods Appl Mech Eng 73:173-189

11. Franca LP, Frey SL, Hughes TJR (1992) Stabilized finite element methods: I. Application to the advective-diffusive model. Comput Methods Appl Mech Eng 95:253-276

12. Hughes TJR, Franca LP, Mallet M (1986) A new finite element formulation for computational fluid dynamics: I. Symmetric forms of the compressible Euler and Navier-Stokes equations and the second law of thermodynamics. Comput Methods Appl Mech Eng 54:223-234

13. El Kadri E. NE (2018) Numerical simulation of compressible viscous flow. IOSR J Mech Civ Eng (IOSR-JMCE) 15(1):44-56

14. El Kadri E. NE (2017) A stable finite element method for compressible flows using enthalpic variables. IOSR J Mech Civ Eng (IOSR-JMCE) 14(6):67-74

15. El Kadri E. NE, Chillal A (2017) Petrov-Galerkin formulation for compressible Euler and Navier-Stokes equations. Adv Sci Technol Eng Syst J (ASTESJ) 2(5):63-69

16. El Kadri E. NE, Soulaïmani A, Deschênes C (2000) A finite element formulation of compressible flows using various sets of independent variables. Comput Methods Appl Mech Eng 181:161-189
17. Weiss JM, Smith WA (1995) Preconditioning applied to variable and constant density flows. AIAA J 33(11):2050-2057

18. Turkel E (1987) Preconditioned methods for solving the incompressible and low speed compressible equations. J Comput Phys 72(2):277-298

19. Guillard H, Murrone A (2004) On the behavior of upwind schemes in the low Mach number limit: II. Godunov type schemes. Comput Fluids 33(4):655-675

20. Yoon SY, Yabe T (1999) The unified simulation for incompressible and compressible flow by the predictor-corrector scheme based on the CIP method. Comput Phys Commun 119(2-3):149-158

21. Ida M (2003) An improved unified solver for compressible and incompressible fluids involving free surfaces. II. Multi-timestep integration and applications. Comput Phys Commun 150(3):300-322

22. Xiao F, Akoh R, li S (2006) Unified formulation for compressible and incompressible flows by using multi-integrated moments II: Multi-dimensional version for compressible and incompressible flows. J Comput Phys 213(1):31-56

23. Harlow FH, Welch JE (1965) Numerical calculation of timedependent viscous incompressible flow of fluid with free surface. Phys Fluids 8(12):2182-2189

24. Chorin AJ (1968) Numerical solution of the Navier-Stokes equations. Math Comput 22(104):745

25. Hauke G, Hughes TJR (1994) A unified approach to compressible and incompressible flows. Comput Methods Appl Mech Eng 113(3-4):389-395

26. Hauke G, Hughes TJR (1998) A comparative study of different sets of variables for solving compressible and incompressible flows. Comput Methods Appl Mech Eng 153(1-2):1-44

27. Polner M, Pesch L, van der Vegt JJW (2007) Construction of stabilization operators for Galerkin least-squares discretizations of compressible and incompressible flows. Comput Methods Appl Mech Eng 196(21-24):2431-2448

28. Pesch L, van der Vegt JJW (2008) A discontinuous Galerkin finite element discretization of the Euler equations for compressible and incompressible fluids. J Comput Phys 227(11):5426-5446

29. Bijl H, Wesseling P (1998) A Unified method for computing incompressible and compressible flows in boundary-fitted coordinates. J Comput Phys 141(2):153-173

30. Caiden R, Fedkiw RP, Anderson C (2001) A numerical method for two-phase flow consisting of separate compressible and incompressible regions. J Comput Phys 166(1):1-27

31. Nithiarasu P, Codina R, Zienkiewicz OC (2006) The characteristicbased split (CBS) scheme-a unified approach to fluid dynamics. Int J Numer Methods Eng 66(10):1514-1546

32. Shakib F, Hughes TJR, Johan Z (1989) A multi group preconditioned GMRES algorithm for nonsymmetric systems arising in finite element analysis. Comput Methods Appl Mech Eng 75:415-465

33. Deschênes C (1984) Modélisation par éléments finis d'un labyrinthe de turbomachine. Thèse de doctorat de I'Institut National Polytechnique de Grenoble

Publisher's Note Springer Nature remains neutral with regard to jurisdictional claims in published maps and institutional affiliations. 